\title{
Revisão Sistemática de Estudos da Psicologia Brasileira sobre Preconceito Racial
}

\author{
Airi M. Sacco ${ }^{1}$ \\ Curso de Psicologia da Universidade Federal de Pelotas, Pelotas, RS, Brasil \\ Maria Clara P. de Paula Couto \\ Instituto de Psiquiatria, Hospital das Clínicas da Faculdade de Medicina da Universidade \\ de São Paulo, São Paulo, SP, Brasil \\ Sílvia H. Koller \\ Departamento de Psicologia do Desenvolvimento e da Personalidade da Universidade \\ Federal do Rio Grande do Sul, Porto Alegre, RS, Brasil
}

\section{Resumo}

O objetivo deste artigo foi realizar uma revisão sistemática sobre os estudos com foco em preconceito racial publicados pela Psicologia brasileira. Foi realizada uma busca nas bases de dados SciELO (Scientific Eletronic Library Online), PePSIC, Index Psi, LILACS (Literatura Latino-Americana e do Caribe em Ciências da Saúde), e PsycINFO, utilizando os termos "racismo" ou "preconceito racial". O banco final incluído na análise foi constituído por 77 artigos, 35 teóricos e 42 empíricos, publicados desde 2001. A Psicologia Social, em suas mais variadas vertentes, foi a base teórica mais utilizada. O tema mais frequente nos artigos empíricos foram as cotas de acesso ao ensino superior. Já os teóricos investigaram temas como as origens do preconceito racial e suas formas de expressão, as consequências do preconceito para as vítimas de racismo, e as influências de teorias raciais no desenvolvimento da Psicologia, entre outros. Foram identificadas algumas lacunas na descrição metodológica dos estudos nacionais, principalmente no que diz respeito à caracterização de participantes, instrumentos e procedimentos adotados. Também foi constatada a necessidade de um incremento na realização de pesquisas que visem a compreender como o preconceito racial se desenvolve em crianças no contexto brasileiro. São escassas, ainda, pesquisas que tenham como foco adultos não-universitários e pessoas que pertençam a grupos discriminados. Além disso, apenas um estudo utilizou medidas implícitas de atitude. $\mathrm{O}$ número relativamente baixo de pesquisas da Psicologia brasileira sobre preconceito racial publicadas, assim como as lacunas identificadas, revelam que, longe de estar saturada, a área ainda tem muito a se desenvolver no país.

Palavras-chave: Preconceito racial, racismo, discriminação, revisão sistemática.

\section{Systematic Review of Brazilian Psychological Studies about Racial Prejudice}

\begin{abstract}
The purpose of this study was to systematically review Brazilian psychological studies focusing on racial prejudice. A search was conducted in the databases SciELO (Scientific Eletronic Library Onli-

Endereço para correspondência: Colegiado de Psicologia, Avenida Duque de Caxias, 250, Pelotas, RS, Brasil, 96030-001. Fone: +55 51 3308-5150. E-mail: amsacco@gmail.com, mariaclara.ppc@gmail.com e silvia. koller@gmail.com

Agência de financiamento: Conselho Nacional de Desenvolvimento Científico e Tecnológico (processos 141660/2011-2 e 474252/2011-6)".
\end{abstract}


ne), PePSIC, Index Psi, LILACS (Literatura Latino-Americana e do Caribe em Ciências da Saúde), and PsycINFO. The final database included in the analysis consisted of 77 articles, 35 theoreticals and 42 empiricals, published since 2001. Social Psychology was the most widely used theorical basis. The affirmative actions, specially the quota system, were the most frequent theme of empirical studies. On the other hand the theoretical studies focused on a variety of topics such as the origins and expression of racial prejudice, the consequences of prejudice for its victims, and the influences of racial theories in the development of Psychology. Results indicated the existence of some methodological gaps in the description of the studies, particularly with respect to the characteristics of participants, instruments and procedures. There was also a lack of studies focusing on the development of prejudice in Brazilian children. In addition further studies are needed with focus on minority groups and adults other than graduate and undergraduate students. Also, only one study has used an implicit measure of attitude. The relatively low number of psychological studies focusing on racial prejudice identified by this review, as well as the significant gaps in the existing research, revealed that is a field of study still in development in Brazil.

Keywords: Racial prejudice, racismo, discrimination, systematic review.

\section{Revisión Sistematica de Estudios de Psicología Brasileña sobre el Prejuicio Racial}

\section{Resumen}

El objetivo de este artículo fue hacer una revisión sistemática de los estudios centrados en prejuicios raciales publicados por la Psicología Brasileña. Se realizó una búsqueda en las bases de datos SciELO (Scientific Eletronic Library Online), PEPSIC, INDEXPsi, LILACS (Literatura Latino-Americana e do Caribe em Ciências da Saúde), y PsycINFO, utilizando los términos "racismo" y "prejuicio racial". La base de datos final incluida en el análisis abarcó 77 artículos publicados desde 2001, 35 teóricos y 42 empíricos. La Psicología Social, en sus diferentes aspectos, ha sido la base teórica más utilizada. El tema más común en los artículos empíricos fue las cuotas de acceso a la educación superior. Por su parte, los artículos teóricos investigaban temas como el origen de los prejuicios raciales y sus formas de expresión, las consecuencias del perjuicio para las víctimas del racismo y la influencia de las teorías raciales en el desarrollo de la Psicología, entre otros temas. Se identificaron algunas carencias en la descripción metodológica de los estudios, particularmente en la caracterización de los participantes, instrumentos y procedimientos adoptados. Además, se encontró la necesidad de incrementar la investigación dirigida a la comprensión de cómo los prejuicios raciales se desarrollan en los niños en el contexto brasileño, investigar a los adultos que no poseen formación universitaria y a las personas pertenecientes a grupos discriminados. Por otra parte, solamente un estudio utilizó mediciones de actitudes implícitas. El número relativamente bajo de investigaciones publicadas, así como las carencias identificadas, revelan que lejos de estar saturada, esta área todavía puede desarrollarse mucho en el país.

Palabras clave: Prejuicio racial, racism, discriminación, revisión sistemática.

O Brasil registra elevados índices de desigualdade social e econômica relacionados à cor da pele (Instituto Brasileiro de Geografia e Estatística [IBGE], 2013). Apesar disso, alguns estudos indicam que muitas pessoas, embora reconheçam a existência de racismo no Brasil, não se consideram preconceituosas (Camino, Silva, Machado, \& Pereira, 2001). O preconceito con- siste em uma avaliação negativa relativa a grupos sociais (Eagly \& Dieckman, 2005) influenciada por vieses no contexto das relações intergrupo (Dovidio \& Gaertner, 2010). No caso específico do preconceito racial, esses grupos sociais são definidos principalmente de acordo com a cor da pele. Em termos conceituais, podemos distinguir ainda preconceito de discriminação. $\mathrm{O}$ 
preconceito está relacionado a questões afetivas, a uma preferência por um grupo em detrimento de outro. A discriminação, por sua vez, consiste em um comportamento. Na discriminação racial, portanto, pessoas que fazem parte de grupos raciais não dominantes (p. ex., pretos e pardos) são tratadas de forma distinta em virtude de sua cor da pele.

O fenômeno de terceirização do preconceito racial é um dos elementos que dificultam a implantação de estratégias efetivas para o combate à discriminação. Na medida em que não se reconhecem como preconceituosas, a tendência é que as pessoas não se sintam parte do público a que são direcionadas campanhas contra o racismo, por exemplo, termo que é utilizado para fazer referência tanto ao preconceito quanto à discriminação. A Psicologia, ciência que estuda o desenvolvimento humano, as relações sociais e a cognição social, para citar apenas algumas áreas, tem potencial não apenas para compreender como essas relações intergrupais se desenvolvem, e como afetam indivíduos e sociedade, mas também para propor intervenções. Já em 2002, no entanto, pesquisadores destacavam a escassez de estudos da Psicologia sobre preconceito racial (Azerêdo, 2002) e a necessidade de a Psicologia refletir sobre sua função social (Oliveira, 2002) como ciência e profissão.

Os estudos sobre preconceito racial realizados na Europa e nos Estados Unidos geralmente envolvem comparações entre dois grupos étnicos ou raciais, tais como brancos e negros (Newheiser \& Olson, 2012), alemães e turcos (Degner \& Wentura, 2011), árabes e judeus (Malkin \& Ari, 2013). O Brasil, contudo, apresenta uma realidade peculiar, visto que no país não existem apenas dois grandes grupos raciais, mas um continuum de cores de pele. Para fins de identificação populacional, o IBGE utiliza cinco grandes grupos: brancos, pardos, pretos, amarelos e indígenas. No Censo Demográfico de 2010, $99 \%$ da população declarou fazer parte de um dos três primeiros grupos (IBGE, 2011; Petruccelli \& Saboia, 2013). No que diz respeito a esses três grandes grupos, a nomenclatura "brancos", "pardos" e "pretos" é aquela adotada pelos órgãos oficiais, mas é apenas uma dentre várias possibilidades.
Algumas organizações preferem utilizar apenas brancos e negros, enquanto a terminologia popular engloba também variações do termo moreno, por exemplo, o que é uma amostra da complexidade da questão racial no Brasil (Petruccelli \& Saboia, 2013; Telles, 2003).

Qual é, então, o papel da Psicologia brasileira no estudo do preconceito racial? Mais do que isso, de que forma a Psicologia tem abordado essa temática no Brasil? Durante a elaboração deste trabalho foi encontrada apenas uma revisão sobre como a Psicologia brasileira tem estudado as relações étnico-raciais (Martins, Santos, \& Colosso, 2013), mas não com foco específico na questão do preconceito. Assim, o objetivo deste artigo foi realizar uma revisão sistemática sobre os estudos com foco em preconceito racial publicados pela Psicologia brasileira. O intuito foi compreender como a Psicologia tem pesquisado esse assunto no Brasil, quais temas estão sendo utilizados para abordar essa questão, que tipo de métodos têm sido adotados, quais são os resultados encontrados, quais são as possíveis lacunas que ainda precisam ser preenchidas e que tipo de contribuição a ciência psicológica tem prestado à sociedade brasileira no que diz respeito ao estudo da temática racial.

\section{Método}

Foi realizada uma busca nas bases de dados SciELO (Scientific Eletronic Library Online), PePSIC, Index Psi, LILACS (Literatura Latino-Americana e do Caribe em Ciências da Saúde), e PsycINFO. Nas quatro primeiras, os termos de busca com operadores booleanos foram "racismo $O R$ preconceito racial". Já na última foram utilizados os termos "(racism OR racial prejudice) AND (Brazil OR Brazilian OR portuguese)". Como o interesse desta revisão foi entender, a partir dos artigos científicos já publicados, como a Psicologia brasileira tem estudado o preconceito racial, optou-se por não delimitar na busca o período de publicação. Assim, para não haver o risco de excluir estudos importantes em virtude apenas da data de sua divulgação, foram incluídos todos os trabalhos publicados até agosto de 2014, quando a busca foi realizada. Da mesma 
forma, o idioma do artigo também não foi utilizado como critério de busca.

Em um primeiro momento, foram excluídos os artigos duplicados entre as bases consultadas. Após, foram aplicados dois critérios de inclusão: ter como tema principal o racismo e estar relacionado à área da Psicologia brasileira. Para checagem do primeiro critério, foram analisados título, resumo e palavras-chave dos artigos. Já para o segundo, foram considerados nome e escopo das revistas, além dos currículos Lattes dos(as) autores(as), nos casos em que as revistas eram multitemáticas ou de outras áreas. Com base nessa análise, foram incluídos: (a) todos os artigos publicados em revistas brasileiras de Psicologia ou multitemáticas em que a Psicologia constasse como uma das áreas do escopo da publicação; e (b) todos os artigos cujo primeiro autor(a) fosse brasileiro(a) e tivesse formação em Psicologia, definida aqui como graduação ou pós-graduação stricto sensu na área. Os textos completos dos artigos selecionados foram recuperados e submetidos a uma nova seleção. Nessa etapa, foram excluídos os artigos que não tinham o texto completo disponível na internet.

Em virtude da padronização limitada nos termos utilizados por pesquisadores, algumas revisões sistemáticas utilizam estratégias complementares para tornar a busca mais compreensiva e abrangente (Costa \& Zoltowski, 2014; DeSousa, Moreno, Gauer, Manfro, \& Koller, 2013; Zoltowski, Costa, Teixeira, \& Koller, 2014). Para ampliar o alcance desta revisão, duas estratégias complementares foram adotadas: pesquisa nas listas de referência dos estudos selecionados previamente e consulta, via e-mail, ao pesquisador com mais estudos publicados na área.

A análise das publicações incluídas nesta revisão levou em consideração a natureza (e.g., trabalho empírico, teórico, etc.) e o tema do estudo, a base teórica, o método, e os resultados encontrados. Todo o processo de seleção dos artigos e extração dos dados foi realizado por duas juízas independentes. Nos dois casos em que houve discordância inicial, foi realizada uma nova análise conjunta até ser encontrado consenso.

\section{Resultados}

A busca inicial nas bases de dados gerou um total de 377 artigos (SciELO: 193, LILACS: 93, Index Psi Periódicos Técnico-Científicos: 66, PePSIC: 25, PsycINFO: 0). Na primeira triagem, foram excluídos 98 trabalhos duplicados entre as bases. Dos 279 artigos restantes, 204 não atenderam aos critérios de inclusão e nove atenderam ao critério de exclusão, ou seja, o texto completo não estava disponível na internet. Restaram, portanto, 66, os quais foram incluídos na análise principal deste estudo. No que diz respeito às estratégias complementares, a busca nas listas de referência teve como resultado a inclusão de 11 novos trabalhos. O pesquisador consultado, por sua vez, considerou o resultado da revisão correto e não fez nenhum acréscimo. Assim, o banco final incluído na análise deste estudo foi constituído por 77 artigos (ver Figura 1), destacados com asterisco na lista de referências. Os resultados serão apresentados de forma descritiva, tendo como base a análise de elementos relativos ao ano de publicação dos trabalhos, periódicos responsáveis pelas publicações, natureza dos estudos, aspectos metodológicos e temáticas abordadas.

A análise dos artigos encontrados revelou que a publicação de trabalhos específicos da Psicologia sobre preconceito racial, dentro dos critérios especificados neste estudo, teve início em 2001. Apesar de terem sido encontrados artigos sobre o tema publicados na década anterior, eles não estavam disponíveis na internet e, portanto, não foram incluídos na revisão. Dos nove estudos não recuperados, quatro eram anteriores a 2001. Desde então, o número de publicações não seguiu um padrão linear ao longo dos anos (ver Figura 2). Em 2013, a área atingiu o número máximo de trabalhos sobre o tema publicados em um único ano (10) e, em 2001 foram apenas três. Em 2014, quatro artigos foram recuperado, mas esse é um número provisório, visto que a busca foi efetuada em meados do ano mencionado. 


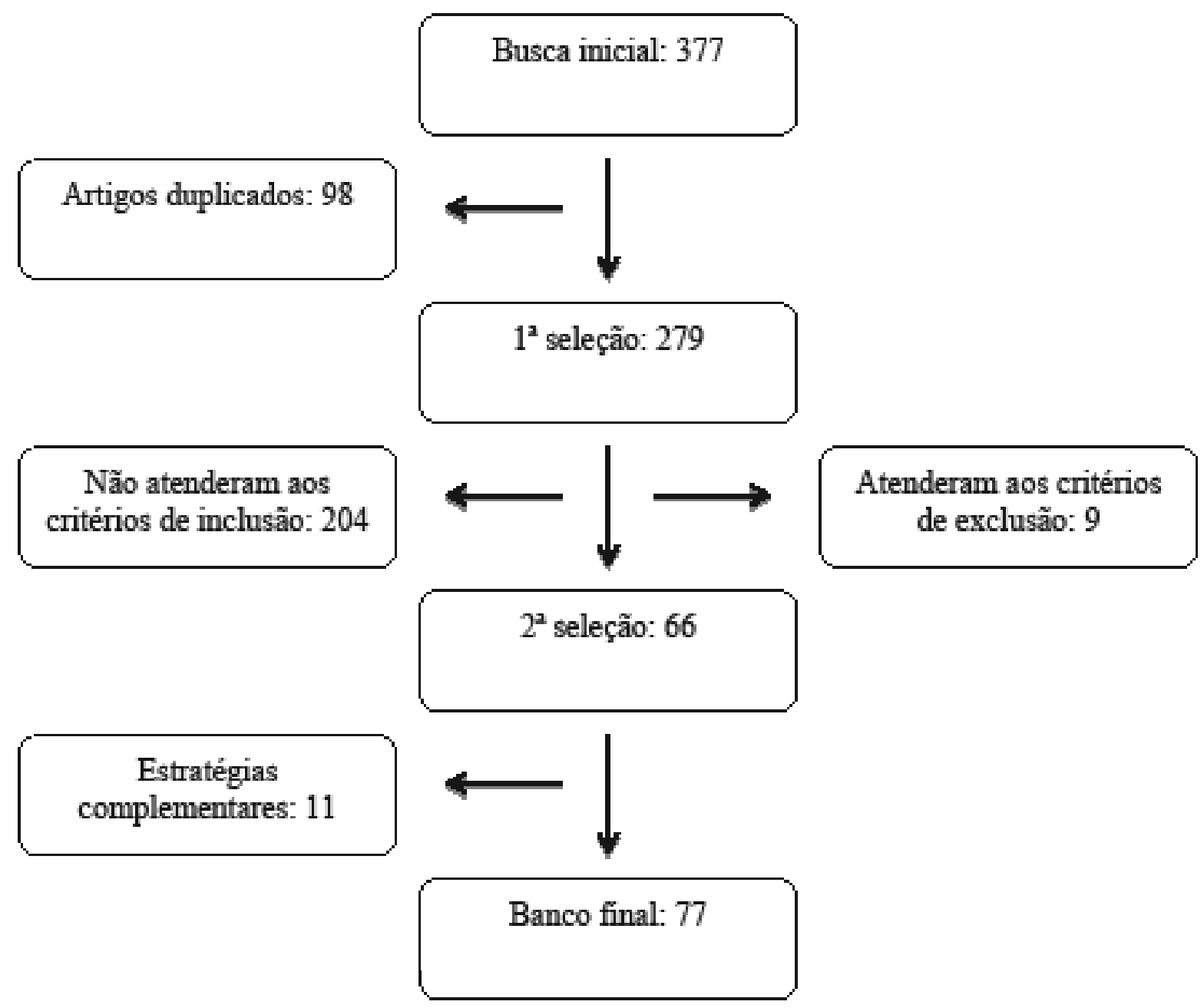

Figura 1. Fluxograma de seleção dos artigos.

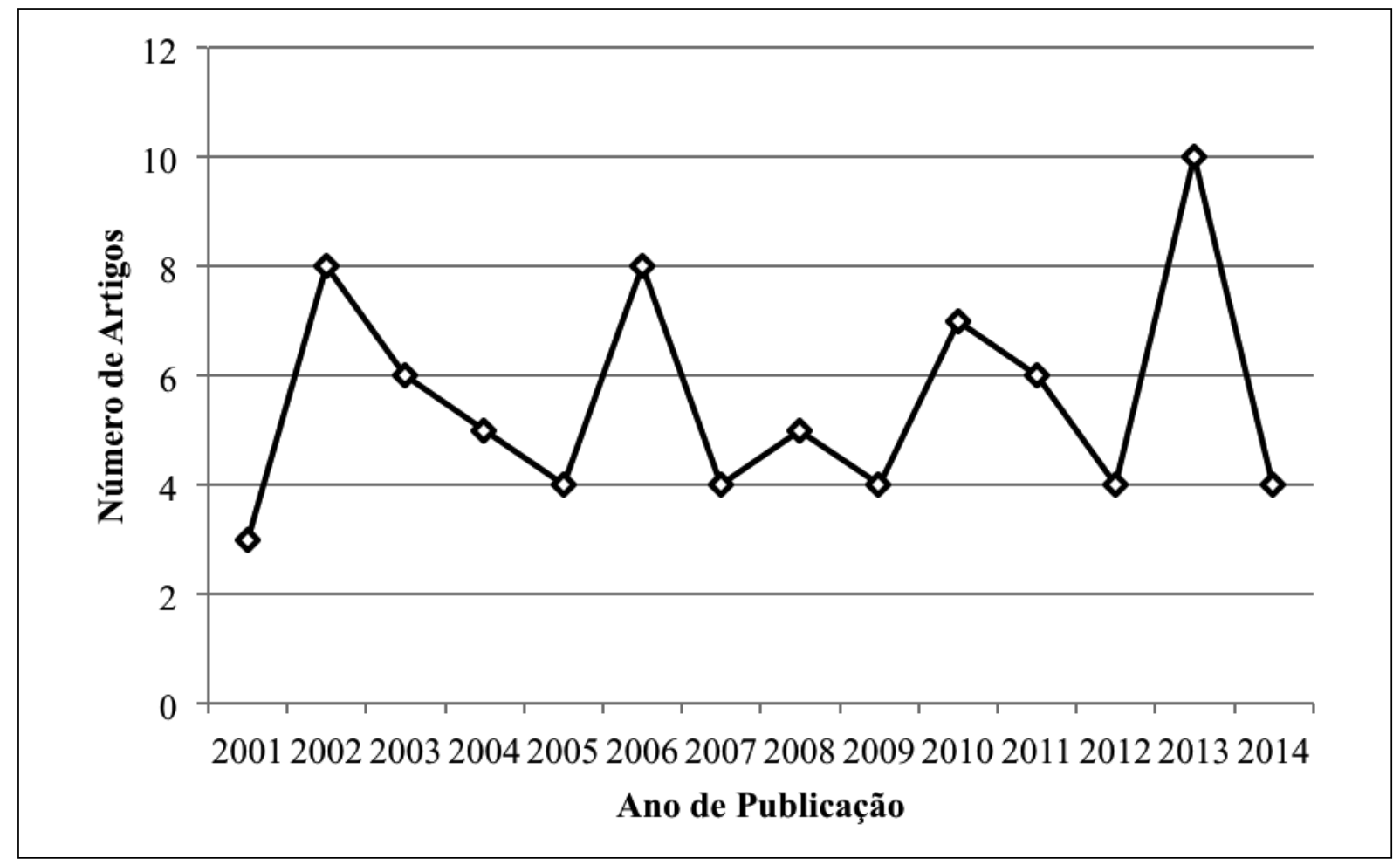

Figura 2. Número de artigos publicados por ano. 
Os 77 trabalhos analisados neste estudo foram publicados em 38 revistas diferentes, 36 das quais são editadas no Brasil e duas no exterior. Com relação ao idioma, três artigos foram publicados em espanhol (Pires, 2010; Pires \& Alonso, 2008; Rottenbacher, Espinosa, \& Manuel Magallanes, 2011), dois em inglês (França \& Lima, 2011; São Paulo, 2010), um em português de Portugal (França \& Monteiro, 2004) e os outros $71 \mathrm{em}$ português brasileiro. A maioria dos periódicos, 27, publicou apenas um artigo sobre o tema (ver Tabela 1). As revistas que mais se destacaram foram Psicologia \& Sociedade (12), Psicologia: Ciência e Profissão (8), e Revista de Psicologia Politica (7). Essas três revistas têm em comum, na definição de suas missões, uma preocupação explícita com a socialização do conhecimento psicológico e com a interface entre a Psicologia e a sociedade. Essa característica pode ajudar a explicar o elevado número de artigos sobre racismo publicados por elas.

\section{Tabela 1}

Número de Artigos Publicados por Revista, com Avaliação segundo o Qualis Periódicos da Capes entre Parênteses

\begin{tabular}{|c|c|c|}
\hline Revistas (Qualis) & Artigos & Referências \\
\hline Análise Psicológica - Portugal (B1) & 1 & França \& Monteiro (2004) \\
\hline Arquivos Brasileiros de Psicologia (A2) & 1 & E. F. Santos \& Scopinho (2011) \\
\hline Avaliação (B1) & 1 & Crisostomo \& Reigota (2010) \\
\hline Eccos Revista Cientifica (B4) & 1 & Ferreira \& Camargo (2001) \\
\hline Educação e Pesquisa (B1) & 2 & $\begin{array}{c}\text { Rosemberg, Bazilli, \& Silva (2003); P. V. B. Silva, } \\
\text { Teixeira, \& Pacifico (2013) }\end{array}$ \\
\hline Estudos Afro-Asiáticos (B1) & 1 & G. A. Santos (2002) \\
\hline Estudos de Psicologia - Campinas (A2) & 1 & P. Oliveira (2009) \\
\hline Estudos de Psicologia - Natal (A2) & 5 & $\begin{array}{l}\text { Faro \& Pereira (2011); Lima \& Vala (2004a); Masiero } \\
\text { (2005); P. Oliveira (2005); Vianna \& Neves (2011) }\end{array}$ \\
\hline Estudos e Pesquisas em Psicologia (B1) & 3 & $\begin{array}{l}\text { Dahia (2010); Naiff, Naiff, \& Souza (2009); Serra \& } \\
\text { Schucman (2012) }\end{array}$ \\
\hline Estudos Psicanalíticos (B4) & 1 & Delfino, Reis, Foscarini, \& Avelino (2006) \\
\hline European Journal of Social Psychology & 1 & França \& Monteiro (2013) \\
\hline Fractal: Revista de Psicologia (B1) & 1 & Zamora (2012) \\
\hline Ide (B4) & 1 & Koltai (2008) \\
\hline Imaginário (B3) & 1 & Scandiucci (2006) \\
\hline Interação em Psicologia (A2) & 1 & Nery \& Conceição (2006) \\
\hline $\begin{array}{c}\text { Intercom: Revista Brasileira de Ciências } \\
\text { da Comunicação (B3) }\end{array}$ & 1 & P. V. B. Silva, Rocha, \& Santos (2012) \\
\hline $\begin{array}{l}\text { International Journal of Conflict } \\
\text { and Violence }(\mathrm{B} 1)\end{array}$ & 1 & França \& Lima (2011) \\
\hline Psico PUCRS (A2) & 1 & Fernandes, Almeida, \& Nascimento (2008) \\
\hline Psico USF (A2) & 2 & $\begin{array}{c}\text { Nery \& Costa (2009); Vasconcelos, Gouveia, Souza, } \\
\text { Sousa, \& Jesus (2004) }\end{array}$ \\
\hline Psicologia: Ciência e Profissão (A2) & 8 & $\begin{array}{l}\text { Azerêdo (2002); Ferreira \& Camargo (2011); Ferreira \& } \\
\text { Mattos (2007); Jesus (2013); Lemos \& Galindo (2013); } \\
\text { Masiero (2002); C. M. Oliveira (2002); S. G. Silva (2003) }\end{array}$ \\
\hline
\end{tabular}




\begin{tabular}{|c|c|c|}
\hline Revistas (Qualis) & Artigos & Referências \\
\hline Psicologia Clínica (B1) & 1 & Fuks (2007) \\
\hline Psicologia e Sociedade (A2) & 12 & $\begin{array}{l}\text { Camino, Gouveia, Maia, Paz, \& Laureano (2013); } \\
\text { Dahia (2013); Camino, Tavares, Torres, Álvaro, } \\
\text { \& Garrido (2014); Ferreira (2002); } \\
\text { Guarnieri \& Melo-Silva (2007); Lins, Lima-Nunes, } \\
\text { \& Camino (2014); A. V. L. Nunes \& Camino (2011); } \\
\text { M. L. P. Oliveira, Meneghel, \& Bernardes (2009); } \\
\text { Pires (2010); Pires \& Alonso (2008); Roso, Strey, } \\
\text { Guareschi, \& Bueno (2002); Schucman (2014) }\end{array}$ \\
\hline Psicologia em Estudo (A2) & 3 & $\begin{array}{c}\text { Chaves (2003a); Guareschi et al. (2002); W. S. Santos, } \\
\text { Gouveia, Navas, Pimente, \& Gusmão (2006) }\end{array}$ \\
\hline Psicologia em Revista (B3) & 1 & Teshainer \& Küller (2005) \\
\hline Psicologia em Revista - Belo Horizonte (B1) & 2 & Reis (2004); Máximo, Larrain, Nunes, \& Lins (2012) \\
\hline Psicologia: Reflexão e Crítica (A1) & 3 & $\begin{array}{l}\text { Fernandes, Da Costa, Camino, \& Mendoza (2007); Lima, } \\
\text { Machado, Ávila, Lima, \& Vala (2006); Pereira, Torres, \& } \\
\text { Almeida (2003) }\end{array}$ \\
\hline Psicologia: Teoria e Pesquisa (A1) & 1 & Lima \& Vala (2004b) \\
\hline Psicologia USP (A2) & 3 & Lima \& Vala (2005); S. S. Nunes (2006); Weyler (2006) \\
\hline $\begin{array}{l}\text { Revista Brasileira de Crescimento e } \\
\text { Desenvolvimento Humano (B1) }\end{array}$ & 1 & Chaves (2003b) \\
\hline Revista Brasileira de Educação (B1) & 1 & Lima, Neves, \& Bacellar e Silva (2014) \\
\hline Revista de Administração Mackenzie (B1) & 1 & São Paulo (2010) \\
\hline Revista de Psicologia Política (B2) & 7 & $\begin{array}{l}\text { Acevedo, Nohara, \& Ramuski (2010); Camino et } \\
\text { al. (2001); P. Oliveira, Santos, \& Soares (2010); } \\
\text { Rottenbacher et al. (2011); Schucman (2010); } \\
\text { M. P. Silva (2001); Vilhena (2006) }\end{array}$ \\
\hline Revista Estudos Feministas (B1) & 1 & Mayorga, Coura, Miralles, \& Cunha (2013) \\
\hline Revista Psicologia e Saúde (B4) & 1 & Duarte \& Roazzi (2013) \\
\hline Revista Psicologia: Teoria e Prática (A2) & 1 & Martins et al. (2013) \\
\hline Saúde em Debate (B2) & 1 & Tavares, Oliveira, \& Lages (2013) \\
\hline Saúde e Sociedade (B2) & 1 & Guimarães \& Podkameni (2008) \\
\hline Sociedade e Estado (B2) & 1 & Dahia (2008) \\
\hline
\end{tabular}

Nota . Capes $=$ Coordenação de Aperfeiçoamento de Pessoal de Nível Superior.

No que diz respeito à classificação das revistas no Qualis Periódicos da última avaliação trienal da Capes, $34,2 \%$ foram classificadas no estrato A, sendo duas A1 e 11 A2, e $65,8 \%$ no estrato $\mathrm{B}$, sendo $13 \mathrm{~B} 1$, quatro $\mathrm{B} 2$, três $\mathrm{B} 3$, e quatro B4. Apesar de pouco mais de dois terços dos periódicos estarem no estrato $\mathrm{B}$, a tendência se modifica quando são analisados o número de artigos publicados por cada revista: 42 foram pu- blicados em periódicos avaliados como A e 34 em periódicos $\mathrm{B}$.

Com relação à natureza dos estudos, 35 são teóricos e 42 empíricos. A Psicologia Social, em uma ampla variedade de vertentes, foi a base teórica mais utilizada, seguida da Psicanálise. No que diz respeito aos artigos empíricos, $50 \%$ utilizaram método quantitativo, $40 \%$ qualitativo e $10 \%$ misto. As informações relativas ao mé- 
todo e ao delineamento dos estudos, no entanto, às vezes precisaram ser inferidas, pois em alguns casos não foram explicitadas pelos(as) autores(as). Quando consideradas as descrições de participantes, instrumentos e procedimentos utilizados, apenas onze dos 42 artigos empíricos analisados apresentaram informações metodológicas completas (a saber: Fernandes et al., 2008; França \& Lima, 2011; França \& Monteiro, 2004, 2013; Lima et al., 2014; Lima \& Vala, 2004b; Máximo et al., 2012; Nery \& Conceição, 2006; M. L. P. Oliveira et al., 2009; W. S. Santos et al., 2006; P. V. B. Silva et al., 2012). Os outros 31 artigos apresentaram falhas na descrição de um, dois ou mesmo três dos itens avaliados, como indica a Tabela 2. Dos 11 artigos com um dos itens incompletos, sete apresentaram alguma falha na descrição dos participantes e não explicitaram dados importantes para a caracterização da amostra, tais como informações relativas a sexo, idade e cor/raça, por exemplo. Em relação a esse aspecto, é interessante destacar que, apesar de ter seu foco no preconceito racial, 11 artigos empíricos analisados não informaram a cor/raça dos participantes de seus estudos (a saber, Camino et al., 2013; Camino et al., 2014; Guareschi et al., 2002; Lins et al., 2014; A. V. L. Nunes \& Camino, 2011; Pereira et al., 2003; Rottenbacher et al., 2011; São Paulo, 2010; M. P. Silva, 2001; Tavares et al., 2013; Vasconcelos et al., 2004), informação essencial para a compreensão dos resultados desse tipo de pesquisa (Camino et al., 2001).

Tabela 2

Artigos que Apresentaram alguma Lacuna na Descrição de Participantes, Instrumentos e/ou Procedimentos

\begin{tabular}{|c|c|c|c|}
\hline \multirow{2}{*}{ Artigos } & \multicolumn{3}{|c|}{ Descrição incompleta } \\
\hline & Participantes & Instrumentos & Procedimentos \\
\hline Camino et al. (2013) & $\mathrm{x}$ & & \\
\hline Camino et al. (2001) & & & $\mathrm{x}$ \\
\hline Camino et al. (2014) & $\mathrm{x}$ & & \\
\hline Crisostomo \& Reigota (2010) & $\mathrm{x}$ & $\mathrm{x}$ & $\mathrm{x}$ \\
\hline Fernandes et al. (2007) & & & $\mathrm{x}$ \\
\hline Ferreira \& Camargo (2011) & $\mathrm{x}$ & $\mathrm{x}$ & $\mathrm{x}$ \\
\hline Ferreira \& Mattos (2007) & & & $\mathrm{x}$ \\
\hline Guareschi et al. (2002) & $\mathrm{x}$ & & $\mathrm{x}$ \\
\hline Guimarães \& Podkameni (2008) & $\mathrm{x}$ & & $\mathrm{x}$ \\
\hline Jesus (2013) & $\mathrm{x}$ & $\mathrm{x}$ & $\mathrm{x}$ \\
\hline Lima et al. (2006) & $\mathrm{x}$ & & \\
\hline Lima \& Vala (2005) & $\mathrm{x}$ & & \\
\hline Lins et al. (2014) & $\mathrm{x}$ & & \\
\hline Naiff et al. (2009) & $\mathrm{x}$ & $\mathrm{x}$ & $\mathrm{x}$ \\
\hline Nery \& Costa (2009) & & $\mathrm{x}$ & $\mathrm{x}$ \\
\hline A. V. L. Nunes \& Camino (2011) & $\mathrm{x}$ & & $\mathrm{x}$ \\
\hline P. Oliveira (2009) & $\mathrm{x}$ & $\mathrm{x}$ & $\mathrm{x}$ \\
\hline P. Oliveira et al. (2010) & $\mathrm{x}$ & $\mathrm{x}$ & $\mathrm{x}$ \\
\hline Pereira et al. (2003) & $\mathrm{x}$ & & $\mathrm{x}$ \\
\hline Pires (2010) & & & $\mathrm{x}$ \\
\hline Pires \& Alonso (2008) & $\mathrm{x}$ & & \\
\hline
\end{tabular}




\begin{tabular}{|c|c|c|c|}
\hline \multirow{2}{*}{ Artigos } & \multicolumn{3}{|c|}{ Descrição incompleta } \\
\hline & Participantes & Instrumentos & Procedimentos \\
\hline Reis (2004) & $\mathrm{x}$ & $\mathrm{x}$ & $\mathrm{x}$ \\
\hline Rottenbacher et al. (2011) & $\mathrm{x}$ & & $\mathrm{x}$ \\
\hline São Paulo (2010) & $\mathrm{x}$ & & $\mathrm{x}$ \\
\hline Schucman (2014) & & $\mathrm{x}$ & $\mathrm{x}$ \\
\hline Serra \& Schucman (2012) & & $\mathrm{x}$ & $\mathrm{x}$ \\
\hline M. P. Silva (2001) & $\mathrm{x}$ & $\mathrm{x}$ & $\mathrm{x}$ \\
\hline P. V. B. Silva et al. (2013) & $\mathrm{x}$ & $\mathrm{x}$ & $\mathrm{x}$ \\
\hline Tavares et al. (2013) & $\mathrm{x}$ & $\mathrm{x}$ & $\mathrm{x}$ \\
\hline Vasconcelos et al. (2004) & $\mathrm{x}$ & & \\
\hline
\end{tabular}

As lacunas na descrição de aspectos metodológicos podem afetar diretamente a viabilidade de, no caso das pesquisas quantitativas, serem realizados estudos de replicação. De acordo com os dados da Tabela 2, 14 dos 21 estudos quantitativos apresentaram algum problema na descrição de participantes, instrumentos e/ou procedimentos. Considerando apenas esses itens e sem entrar, portanto, na análise dos dados, apenas sete estudos quantitativos poderiam ser replicados unicamente com as informações que constavam nos artigos ou com eventuais solicitações de instrumentos. A replicação dos outros 14 poderia ser prejudicada pela insuficiência de informações metodológicas.

Embora não se possa falar em replicação no caso dos estudos qualitativos ou mistos, a análise das informações metodológicas desses estudos também revelou uma série de lacunas. Nove estudos qualitativos e um misto apresentaram descrições incompletas de todos os três aspectos metodológicos avaliados, o que dificultaria que fossem utilizados como base para a elaboração de estudos semelhantes. Dos 42 artigos empíricos analisados, portanto, $30(71,4 \%)$ apresentaram alguma lacuna na descrição do método (ver Tabela 2).

No que diz respeito aos instrumentos, os estudos qualitativos utilizaram predominantemente entrevistas, presente em $80 \%$ deles. Já os quantitativos empregaram fundamentalmente questionários e escalas (89\%). Grande parte dos questionários foi produzida especificamente para as pesquisas nas quais foi utilizada. As escalas, por sua vez, ou já eram validadas ou foram adaptadas de outros estudos. A mais aplicada, presente em quatro publicações, foi a Escala de Racismo Moderno (McConahay, Hardee, \& Batts, 1981). No entanto, as versões utilizadas variaram, visto que três dos quatro estudos fizeram suas próprias adaptações do instrumento para o Brasil (Pires \& Alonso, 2008; W. S. Santos et al., 2006; São Paulo, 2010) e um utilizou uma adaptação espanhola da escala (Pires, 2010).

Todos os estudos empíricos adotaram recortes transversais e apenas quatro indicaram de alguma forma a utilização de delineamento ou procedimentos experimentais (França \& Monteiro, 2013; Lima et al., 2006; Lima \& Vala, 2004b; Pereira et al., 2003). Esses foram também os que mais se assemelharam metodologicamente aos trabalhos publicados em periódicos internacionais, que, na investigação de preferências raciais, primam pela utilização de delineamentos experimentais e, nos últimos anos, de medidas implícitas, nas quais os participantes não são questionados diretamente sobre suas preferências (Raabe \& Beelmann, 2011). No caso do Brasil, somente um dos estudos selecionados (Lima et al., 2006) utilizou uma medida indireta de atitude, o Teste de Associação Implícita (IAT; Greenwald, McGhee, \& Schwartz, 1998), para investigar o preconceito automático. Todos os outros artigos empregaram exclusivamente medidas explícitas (i.e., medidas de autorelato tais como questionários e escalas). 
Ainda em relação ao método, em 32 dos 42 estudos empíricos, os participantes eram adultos e, em $65,6 \%$ desses casos, as pesquisas foram realizadas com estudantes universitários. As crianças foram o foco de apenas seis estudos. Os instrumentos utilizados com essa população foram fundamentalmente entrevistas, análise de desenhos e tarefas de alocação de recursos. Em nove estudos empíricos os participantes foram indivíduos ou grupos vítimas de preconceito racial (neste caso, pessoas pardas ou pretas). Em outros 26, os participantes foram pessoas brancas.

No que diz respeito à temática dos estudos, a partir de 2004 começaram a ser publicados trabalhos com foco nas políticas afirmativas, especialmente nas cotas de acesso ao ensino superior. Treze artigos empíricos incluídos na revisão tiveram como tema as cotas, sendo oito deles publicados a partir de 2009. Dez dessas publicações investigaram, de alguma forma, as atitudes explícitas dos participantes frente ao sistema de reserva de vagas nas universidades. Em seis, a maioria dos participantes se posicionou de maneira desfavorável às cotas (Camino et al., 2013; Camino et al., 2014; Lima et al., 2014; Naiff et al., 2009; P. Oliveira, 2009; São Paulo, 2010). Em outras duas, que incluíram a realização de sociodramas, foram identificadas indiferença, descaso e desqualificação em relação à questão racial (Nery \& Costa, 2009) e atitudes raciais discriminatórias (Nery \& Conceição, 2006) por parte de estudantes não cotistas.

No estudo realizado por Camino e colaboradores (2013), os resultados indicaram que há uma relação entre o discurso sobre as desigualdades raciais e a posição frente à política de cotas. Todos os participantes que acreditavam haver uma diferença genética entre brancos e pretos se posicionaram contra as cotas. Já aqueles que relacionavam as desigualdades socioeconômicas entre os grupos raciais a questões históricas, foram majoritariamente favoráveis a essa política. Um grupo intermediário foi constituído por aqueles que se apoiavam no discurso miscigenatório para justificar que não há diferenças entre brancos e pretos no Brasil: $42 \%$ se posicionaram a favor e $58 \%$ contra as cotas.
Três estudos contaram com a participação de pessoas potencialmente beneficiadas pelo sistema de reserva de vagas. Nesses estudos foram identificadas preocupação com o bom desempenho acadêmico para minimizar a discriminação sofrida na universidade (Nery \& Costa, 2009), receio de que as cotas gerem ainda mais preconceito contra os negros (Crisostomo \& Reigota, 2010) e dificuldades relacionadas à definição de quem é negro no Brasil, o que poderia ser um problema para a implantação dessa política (Reis, 2004).

Apesar de prevalente nos estudos empíricos, o tema das cotas de acesso ao ensino superior foi abordado em apenas um dos artigos teóricos, que consistiu em uma revisão sobre o debate acerca das ações afirmativas no Brasil (Guarnieri \& Melo-Silva, 2007). Os principais tópicos dos ensaios teóricos foram: as origens do racismo e suas formas de expressão (Fuks, 2007; Koltai, 2008; Lima \& Vala, 2004a; S. S. Nunes, 2006; C. M. Oliveira, 2002; G. A. Santos, 2002; Schucman, 2010; S. G. Silva, 2003; Teshainer \& Küller, 2005; Vianna \& Neves, 2011), as influências do preconceito para as vítimas de racismo (Faro \& Pereira, 2011; Ferreira, 2002; Ferreira \& Camargo, 2001; E. F. Santos \& Scopinho, 2011; Vilhena, 2006; Zamora, 2012), as influências de teorias raciais no desenvolvimento da Psicologia (Chaves, 2003b; Masiero, 2002, 2005; Weyler, 2006), o desenvolvimento do racismo (Chaves, 2003a; Dahia, 2013; Duarte \& Roazzi, 2013), os discursos raciais na mídia e em livros didáticos (Acevedo et al., 2010; Rosemberg et al., 2003; Roso et al., 2002), e a análise do riso como forma de expressão do preconceito racial (Dahia, 2008, 2010). Outros assuntos abordados foram a violência contra povos indígenas (Lemos \& Galindo, 2013), as relações entre colonialismo, racismo e questões de gênero (Mayorga et al., 2013), a cultura do hip hop (Scandiucci, 2006), a análise de uma peça teatral (Delfino et al., 2006), o papel da dimensão política na formação em Psicologia (Azerêdo, 2002), e as publicações da Psicologia sobre relações étnico-raciais (Martins, Santos, \& Colosso, 2013).

Dos 35 artigos teóricos analisados, 15 trataram o preconceito racial como uma questão 
relacionada a estratégias de poder e dominação social. Alguns desses estudos destacaram que o desequilíbrio de poder entre brancos e negros parece estar relacionado a uma disparidade social e econômica (Acevedo et al., 2010; Dahia, 2013). Essas estratégias de dominação visariam a negar os direitos de parte significativa da população e, assim, estabelecer um sistema de exploração mais eficiente (Vilhena, 2006).

Outra questão comum nos estudos teóricos foi a ressalva, feita em dez artigos, de que, em contraste com a importância do assunto, a Psicologia aborda pouco o tema do preconceito racial (Zamora, 2012). Para Chaves (2003b), essa lacuna estaria relacionada à constituição da Psicologia como área de estudo no Brasil. As influências de teorias eugênicas e do evolucionismo social teriam dado origem a uma "Psicologia branca" (p. 36), que teria apenas os brancos como tema de pesquisa (Chaves, 2003b) e estaria a serviço de uma ideologia dominante pautada em uma falsa ideia de democracia racial (Azerêdo, 2002; C. M. Oliveira, 2002). Contudo, de acordo com as autoras do estudo que revisou as publicações da Psicologia sobre relações étnico-raciais, apesar da pequena produção na área, a Psicologia tem colaborado para o aprofundamento da compreensão de fenômenos como o preconceito e a discriminação racial, a influência do branqueamento sobre negros e brancos, e a efetividade das políticas públicas direcionadas a questões étnico-raciais (Martins et al., 2013).

Ainda com relação ao papel da Psicologia nos estudos sobre preconceito racial, Azerêdo (2002) apresentou uma crítica sobre o domínio da Psicologia Social norte-americana na Psicologia Social brasileira e uma suposta desconsideração do contexto histórico por parte dessa disciplina. É curioso constatar, no entanto, que apenas um dos 35 estudos teóricos analisados nesta revisão envolveu a análise de teorias provenientes da Psicologia Social norte-americana (a saber, Lima \& Vala, 2004a). A maioria apoiou-se em autores da filosofia, sociologia e antropologia européias, como Michel Foucault e Hanna Arendt, por exemplo, além da Psicanálise. O mesmo resultado foi identificado na revisão sobre os estudos focados nas relações étnico-raciais (Mar- tins et al., 2013): a maioria dos trabalhos utilizou a Psicologia Social como referencial teórico, com ênfase na Teoria Crítica.

\section{Discussão}

Considerando a relevância social do estudo sobre preconceito racial no Brasil e as contribuições que a Psicologia potencialmente pode gerar, a quantidade de artigos publicados sobre o tema no país ainda é restrita. Dos 279 artigos não duplicados encontrados inicialmente, apenas 66 eram realmente da Psicologia e diziam respeito ao preconceito racial. Tendo em vista os 77 artigos analisados, contudo, mais da metade foi publicada em periódicos Qualis A, o que pode ser um indicativo de que o preconceito racial está entre a gama de temas que interessa às revistas melhor avaliadas.

Apesar de, a partir dos dados coletados, não ser possível identificar uma tendência no número de artigos sobre o tema publicados por ano, é provável que esse número aumente nos próximos anos. $\mathrm{O}$ preconceito racial está em voga tanto em virtude da frequente divulgação de casos de racismo em diversos setores da sociedade quanto da implantação de políticas de ação afirmativa. A Lei Federal n. 12.711 (2012), por exemplo, determinou um prazo de quatro anos para que as universidades federais reservem $50 \%$ de suas vagas para estudantes que se enquadrem nos critérios sociais e raciais estabelecidos. Dentre os critérios sociais estão: todos os candidatos devem ter cursado o ensino médio em escolas públicas e metade precisa ter renda familiar de até 1,5 salário mínimo per capita. Já os critérios raciais compreendem a seleção de candidatos autodeclarados pretos, pardos e indígenas, em proporção no mínimo igual àquela presente na população do estado no qual está localizada a instituição em questão.

De acordo com alguns dos estudos analisados neste artigo, a opinião da população sobre o tema da reserva de vagas no ensino superior é polarizada, especialmente no que diz respeito aos critérios raciais. A polêmica em torno deste tópico possivelmente gerará o desenvolvimento de mais pesquisas para que o assunto seja 
compreendido em sua totalidade. $\mathrm{O}$ aumento na quantidade de publicações, no entanto, precisa ser acompanhado também de um incremento na qualidade dos estudos. O fato de mais de $70 \%$ dos trabalhos empíricos apresentarem alguma lacuna na descrição metodológica indica a necessidade da adoção de maior rigor por parte da comunidade acadêmica tanto na execução dos estudos quanto na descrição dos procedimentos adotados. A exposição clara e objetiva dos passos metodológicos de uma pesquisa é fundamental para que os leitores compreendam o que foi realizado e possam contextualizar os resultados encontrados. Também é condição sine qua non para que outros pesquisadores consigam trabalhar em estudos de replicação ou mesmo comparar dados de diferentes pesquisas.

Os recentes casos de fraude em publicações da Psicologia (Callaway, 2011) foram disparadores para que sociedades internacionais, principalmente ligadas à Psicologia Social, incentivassem a realização de estudos de replicação (Devine, 2012). A iniciativa Many Labs, por exemplo, reúne pesquisadores de todo o mundo, com o intuito de replicar efeitos encontrados nos mais variados estudos (Klein et al., 2014). Esse tipo de pesquisa, contudo, só é viável quando os métodos são bem descritos.

Ainda com relação ao método dos estudos, esta revisão identificou predominância da utilização de medidas explícitas para avaliação do preconceito. $\mathrm{O}$ emprego desse tipo de medida para investigação de temáticas polêmicas, como no caso do preconceito racial, tem sido questionado por permitir que o participante controle as suas respostas, de modo a se apresentar de forma socialmente desejável (Brauer, Wasel, \& Niedenthal, 2000; De Houwer, 2003; Fazio, Jackson, Dunton, \& Williams, 1995; Greenwald \& Banaji, 1995). Em virtude disso, no cenário internacional há uma tendência à utilização de medidas implícitas de atitude, as quais são automáticas e não são passíveis de controle voluntário (Fazio \& Olson, 2003; Olson \& Fazio, 2009; Sritharan \& Gawronski, 2010). Chama a atenção, portanto, que apenas um estudo incluído nesta revisão tenha utilizado uma medida implícita (ver Lima et al., 2006).
Nessa mesma linha, poucos estudos utilizaram delineamentos experimentais. Por oferecer um maior controle sobre as variáveis envolvidas no estudo, a adoção de delineamentos experimentais ou quase-experimentais oferece um maior poder explicativo aos pesquisadores. Tanto a área da Psicologia Social Experimental quanto a Psicologia do Desenvolvimento foram responsáveis por um número incipiente de artigos sobre preconceito racial identificados nesta revisão. Esse fenômeno é interessante porque tanto uma área quanto a outra têm muito a contribuir com o estudo sobre esse tema. No Brasil parece existir uma certa desavença entre diferentes áreas da Psicologia, o que pode ser prejudicial para o avanço do conhecimento.

O editoral do volume especial do European Journal of Social Psychology sobre as origens do viés intergrupo, publicado em 2010, indicou a importância de áreas como a Psicologia Social, a Psicologia Experimental e a Psicologia do Desenvolvimento consultarem umas às outras e desenvolverem estudos que se complementem (Dunham \& Degner, 2010). No caso específico do preconceito racial, essa conexão entre as áreas é fundamental para a compreensão do fenômeno de uma maneira completa, que envolva desde elementos históricos e contextuais até o funcionamento dos processos psicológicos que atuam no desenvolvimento das atitudes raciais explícitas e implícitas.

Na Europa e nos Estados Unidos, muitas pesquisas têm buscado compreender o desenvolvimento das preferências raciais em crianças (Raabe \& Beelmann, 2011). No Brasil, contudo, a maior parte das pesquisas sobre preconceito racial conduzidas pela Psicologia é desenvolvida primordialmente com adultos, mais especificamente com estudantes universitários (em sua maioria, brancos), um público restrito e específico, que corresponde à uma parcela ínfima da população. Há uma série de lacunas, portanto, na realização dos estudos nacionais. Seria interessante haver um incremento na realização de pesquisas que visem a compreender como o preconceito racial se desenvolve em crianças no contexto brasileiro, bem como estudos que 
tenham como foco adultos não-universitários e também pessoas que pertençam a grupos discriminados, por exemplo.

É preciso considerar, contudo, que os resultados obtidos por esta revisão indicam que os estudos sobre preconceito racial realizados pela Psicologia brasileira são recentes e que essa área está em desenvolvimento no país. Em virtude disso, as pesquisas nacionais ainda estão pouco inseridas no debate internacional, tendência que pode ser revertida em um futuro próximo. Como explicitado anteriormente, o Brasil apresenta uma realidade peculiar no que diz respeito à composição racial de sua população, algo que o diferencia de outros contextos e que desperta interesse contínuo da comunidade científica. $\mathrm{O}$ país tem potencial, portanto, para desenvolver pesquisas originais, que contribuam significativamente para o estudo do preconceito racial não apenas a nível local, mas também internacional.

\section{Conclusão}

Esta revisão sistemática teve como objetivo analisar os estudos que a Psicologia brasileira tem publicado sobre preconceito racial no país. Não foi surpresa notar que os periódicos científicos que mais publicaram trabalhos sobre essa temática foram aqueles nos quais há uma preocupação explícita com a interface da Psicologia com a sociedade. A discriminação racial é um grave problema no Brasil e tem suas raízes no preconceito contra determinados grupos sociais. A Psicologia, enquanto ciência que estuda processos sociais, desenvolvimentais e cognitivos, tem potencial para desenvolver estudos que contribuam efetivamente para a compreensão deste fenômeno.

O número relativamente baixo de pesquisas publicadas, assim como as falhas metodológicas identificadas, revelam que, longe de estar saturada, a área ainda tem muito a se desenvolver no país. Tanto a constituição racial da população brasileira quanto os modos como o preconceito racial se manifesta no país são peculiares (Telles, 2003) e, por isso, merecem atenção especial dos estudos psicológicos.
Esta revisão sistemática apresenta algumas limitações, como o fato de ter sido restrita a artigos científicos. Não foram incluídos teses, dissertações e livros, por exemplo. A busca complementar nas referências indicou que muitos estudos se apoiaram nesses outros tipos de publicação, os quais não foram considerados nesta análise. Para além disso, é possível que os descritores utilizados não abranjam alguns trabalhos. Alguns estudos sobre políticas afirmativas, por exemplo, podem ter versado sobre o tema sem necessariamente mencionar as palavras racismo ou preconceito racial e, nesse caso, não terem sido identificados na busca. Essas limitações, no entanto, não invalidam os resultados encontrados ou a relevância do estudo. Ao identificar o foco que estudos de Psicologia têm adotado para investigar o preconceito racial, as autoras esperam que esta revisão seja uma contribuição para aqueles que estão planejando o desenvolvimento de pesquisas nesta área.

\section{Referências}

*Acevedo, C. R., Nohara, J., \& Ramuski, C. L. (2010). Relações raciais na mídia: Um estudo no contexto brasileiro. Revista Psicologia Política, 10(19), 57-73.

*Azerêdo, S. M. M. (2002). O político, o público e a alteridade como desafios para a psicologia. Psicologia: Ciência e Profissão, 22(4), 14-23. doi:10.1590/S1414-98932002000400003

Brauer, M., Wasel, W., \& Niedenthal, P. (2000). Implicit and explicit components of prejudice. Review of General Psychology, 4(1), 79-101. doi:10.1037//1089-2680.4.1.79

Callaway, E. (2011). Report finds massive fraud at Dutch universities. Nature, 479, 15. doi:10.1038/479015a

*Camino, L., Gouveia, L., Maia, L. M., Paz, M. M. A., \& Laureano, M. (2013). Repertórios discursivos contemporâneos sobre as desigualdades raciais no Brasil: Um estudo com estudantes paraibanos de pós-graduação. Psicologia e Sociedade, 25(1), 113-122. doi:10.1590/S010271822013000100013

*Camino, L., Silva, P., Machado, A., \& Pereira, C. (2001). A face oculta do racismo no Brasil: Uma análise psicossociológica. Revista Psicologia Política, 1(1), 13-36. 
*Camino, L., Tavares, T. L., Torres, A. R. R., Álvaro, J. L., \& Garrido, A. (2014). Repertórios discursivos de estudantes universitários sobre cotas raciais nas universidades brasileiras [Número especial]. Psicologia e Sociedade, 26, 117-128. doi:10.1590/S0102-71822014000500013

*Chaves, E. S. (2003a). Nina Rodrigues: Sua interpretação do evolucionismo social e da psicologia das massas nos primórdios da psicologia social brasileira. Psicologia em Estudo, 8(2), 29-37. doi:10.1590/S1413-73722003000200004

*Chaves, E. S. (2003b). O racismo contra o negro e a aprendizagem cultural. Revista Brasileira de Crescimento e Desenvolvimento Humano, 13(2), 1-11.

Costa, A. B. C., \& Zoltowski, A. P. C. (2014). Como escrever um artigo de revisão sistemática. In $\mathrm{S}$. H. Koller, M. C. P. de Paula Couto, \& J. Hohendorff (Eds.), Manual de produção científica (pp. 55-70). Porto Alegre, RS: Grupo A.

*Crisostomo, M. A. S., \& Reigota, M. A. S. (2010). Professoras universitárias negras: Trajetórias e narrativas. Avaliação (Campinas), 15(2), 93106. doi:10.1590/S1414-40772010000200005

*Dahia, S. L. M. (2008). A mediação do riso na expressão e consolidação racismo no Brasil. Sociedade e Estado, 23(3), 697-720.

*Dahia, S. L. M. (2010). Riso: Uma solução intermediária para os racistas no Brasil. Estudos e Pesquisas em Psicologia, 10(2), 373-389.

*Dahia, S. L. M. (2013). Racismo como verbalismo? Delineamentos para compreensão da aquisição do racismo entre cegos congênitos. Psicologia e Sociedade, 25(1), 103-112. doi:10.1590/S010271822013000100012

De Houwer, J. (2003). A structural analysis of indirect measures of attitudes. In J. Musch \& K. C. Klauer (Eds.), The psychology of evaluation: Affective processes in cognition and emotion (pp. 219-244). Mahwah, NJ: Lawrence Erlbaum.

Degner, J., \& Wentura, D. (2011). Types of automatically activated prejudice: Assessing possessorversus other-relevant valence in the evaluative priming task. Social Cognition, 29(2), 182-209. doi:10.1521/soco.2011.29.2.182

*Delfino, E. M., Reis, J. T., Filho, Foscarini, S. R. G., \& Avelino, W. (2006). Anjo negro: Gozo da cor. Estudos Psicanalíticos, 29, 89-93.

DeSousa, D. A., Moreno, A. L., Gauer, G., Manfro, G. G., \& Koller, S. H. (2013). Revisão sistemá- tica de instrumentos para avaliação de ansiedade na população brasileira. Avaliação Psicológica, 12(3), 397-410.

Devine, P. (2012). Open letter to the SPSP membership regarding ethical conduct on research. Dialogue: The official Newsletter of the Society for Personality and Social Psychology, 26(2), 2-3.

Dovidio, J. F., \& Gaertner, S. L. (2010). Intergroup bias. In S. T. Fiske, D. T. Gilbert, \& G. Lindzey (Eds.), Handbook of social psychology ( $5^{\text {th }} \mathrm{ed}$., Vol. 2, pp. 1084-1121). New York: Wiley.

*Duarte, R. O., \& Roazzi, A. (2013). Do canônico e do hegemônico na apropriação do preconceito racial em crianças. Revista Psicologia e Saúde, 5(2), 118-126.

Dunham, Y., \& Degner, J. (2010). Origins of intergroup bias: Developmental and social cognitive research on intergroup attitudes. European Journal of Social Psychology, 40, 563-568. doi:10.1002/ejsp.758

Eagly, A. H., \& Diekman, A. B. (2005). What is the problem? Prejudice as an attitude-in-context. In J. F. Dovidio, P. Glick, \& L. Rudman (Eds.), On the nature of prejudice: Fifty years after Allport (pp. 19-35). Malden, MA: Blackwell.

*Faro, A., \& Pereira, M. E. (2011). Raça, racismo e saúde: A desigualdade social da distribuição do estresse. Estudos de Psicologia (Natal), 16(3), 271-278. doi:10.1590/S1413$-294 X 2011000300009$

Fazio, R. H., Jackson, J. R., Dunton, B. C., \& Williams, C. J. (1995). Variability in automatic activation as an unobstrusive measure of racial attitudes: A bona fide pipeline? Journal of Personality and Social Psychology, 69, 1013-1027. doi:10.1037/0022-3514.69.6.1013

Fazio, R. H., \& Olson, M. A. (2003). Implicit measures in social cognition research: Their meaning and use. Annual Review of Psychology, 54, 297-327. doi:10.1146/annurev.psych.54.101601.145225

*Fernandes, S. C. S., Almeida, S. S. M., \& Nascimento, C. B. S. (2008). Análise do preconceito racial em uma amostra de crianças brancas de 5 a 8 anos de idade. Psico (Porto Alegre), 39(4), 441-447.

*Fernandes, S., Da Costa, J., Camino, L., \& Mendoza, R. (2007). Valores psicossociais e orientação à dominância social: Um estudo acerca do preconceito. Psicologia: Reflexão e Crítica, 20(3), 490498. doi:10.1590/S0102-79722007000300017 
*Ferreira, R. F. (2002). O brasileiro, o racismo silencioso e a emancipação do afro- descendente. Psicologia e Sociedade, 14(1), 69-86. doi:10.1590/ S0102-71822002000100005

*Ferreira, R. F., \& Camargo, A. C. (2001). A naturalização do preconceito na formação da identidade do afro-descendente. Eccos Revista Cientifica, 3(1), 75-92.

*Ferreira, R. F., \& Camargo, A. C. (2011). As relações cotidianas e a construção da identidade negra. Psicologia: Ciência e Profissão, 31(2), 374-389.

*Ferreira, R. F., \& Mattos, R. M. (2007). O afro-brasileiro e o sistema de cotas: Um enfoque psicossocial. Psicologia: Ciência e Profissão, 27(1), 46-63.

*França, D. X., \& Lima, M. E. O. (2011). Affirmative action and ethnic identity in black and indigenous Brazilian children. International Journal of Conflict and Violence, 5(1), 200-210.

*França, D. X., \& Monteiro, M. B. (2004). A expressão das formas indirectas de racismo na infância. Análise Psicológica, 4(22), 705-720.

*França, D. X., \& Monteiro, M. B. (2013). Social norms and the expression of prejudice: The development of aversive racism in childhood. European Journal of Social Psychology, 43(4), 263-271. doi:10.1002/ejsp.1965

*Fuks, B. B. (2007). O pensamento freudiano sobre a intolerância. Psicologia Clínica, 19(1), 59-73. doi:10.1590/S0103-56652007000100005

Greenwald, A. G., \& Banaji, M. R. (1995). Implicit social cognition: Attitudes, self-esteem, and stereotypes. Psychological Review, 102, 4-27.

Greenwald, A. G., McGhee, D. E., \& Schwarz, L. K. (1998). Measuring individual differences in implicit cognition: The Implicit Association Test. Journal of Personality and Social Psychology, 74(6), 1464-1480.

*Guareschi, N., Oliveira, F. P., Giannechini, L. G., Comunello, L. N., Pacheco, L., \& Nardini, M. (2002). As relações raciais na construção das identidades. Psicologia em Estudo, 7(2), 55-64. doi:10.1590/S1413-73722002000200007

*Guarnieri, F. V., \& Melo-Silva, L. L. (2007). Ações afirmativas na educação superior: Rumos da discussão nos últimos cinco anos. Psicologia e Sociedade, 19(2), 70-78. doi:10.1590/S010271822007000200010
*Guimarães, M. A. C., \& Podkameni, A. B. (2008). A rede de sustentação coletiva, espaço potencial e resgate identitário: Projeto mãe-criadeira. Saúde e Sociedade, 17(1), 117-130. doi:10.1590/ S0104-12902008000100011

Instituto Brasileiro de Geografia e Estatística. (2011). Resultados preliminares do censo demográfico 2010. Recuperado em http://ibge.gov.br/home/ estatistica/populacao/censo2010/resultados_ preliminares/default_resultados_preliminares. shtm

Instituto Brasileiro de Geografia e Estatística. (2013). Sintese de indicadores sociais: Uma análise das condições de vida da população brasileira 2013. Rio de Janeiro, RJ: Autor.

*Jesus, J. G. (2013). O desafio da convivência: Assessoria de diversidade e apoio aos cotistas (2004-2008). Psicologia: Ciência e Profissão, $33(1), 222-233$.

Klein, R. A., Ratliff, K. A., Vianello, M., Adams, R. B., Jr., Bahník, S., Bernstein, M. J., \& Nosek, B. A. (2014). Data from investigating variation in replicability: A "Many Labs" replication project. Journal of Open Psychology Data, 2(1), e4. doi:10.5334/jopd.ad

*Koltai, C. (2008). Racismo: Uma questão cada vez mais delicada. Ide, 31(47), 66-69.

Lei Federal n. 12.711. (2012, 30 ago.). Diário Oficial da União, Seção 1, p. 1. Recuperado em http://www.planalto.gov.br/ccivil_03/_ato20112014/2012/lei/112711.htm

*Lemos, F. C. S., \& Galindo, D. C. G. (2013). Massacre e resistência Kaiowá e Guarani: Interrogações às Psicologias nos traçados do intolerável. Psicologia: Ciência e Profissão, 33(4), 976-987.

*Lima, M. E. O., Machado, C., Ávila, J., Lima, C., \& Vala, J. (2006). Normas sociais e preconceito: O impacto da igualdade e da competição no preconceito automático contra os negros. Psicologia: Reflexão e Crítica, 19(2), 309-319.

*Lima, M. E. O., Neves, P. S. C., \& Bacellar e Silva, P. (2014). A implantação de cotas na universidade: Paternalismo e amaça à posição dos grupos dominantes. Revista Brasileira de Educação, 19(56), 141-163. doi:10.1590/S141324782014000100008

*Lima, M. E. O., \& Vala, J. (2004a). As novas formas de expressão do preconceito e do racismo. Estudos de Psicologia (Natal), 9(3), 401-411. doi:10.1590/S1413-294X2004000300002 
*Lima, M. E. O., \& Vala, J. (2004b). Sucesso social, branqueamento e racismo. Psicologia, Teoria e Pesquisa, 20(1), 11-19. doi:10.1590/S010237722004000100003

*Lima, M. E. O., \& Vala, J. (2005). A cor do sucesso: Efeitos da performance social e econômica no branqueamento e na infra-humanização dos negros no Brasil. Psicologia USP, 16(3), 143-165. doi:10.1590/S0103-65642005000200008

*Lins, S. L. B., Lima-Nunes, A., \& Camino, L. (2014). O papel dos valores sociais e variáveis psicossociais no preconceito racial brasileiro. Psicologia e Sociedade, 26(1), 95-105. doi:10.1590/S010271822014000100011

McConahay, J. B., Hardee, B. B., \& Batts, V. (1981). Has racism declined in America? It depends upon who is asking and what is asked. Journal of Conflict Resolution, 25, 563-579.

Malkin, G., \& Ari, R. B. (2013). Prejudice and "Vladimir's choice" among Israeli arabs and jews: Symmetrical and asymmetrical trends. Group Processes \& Intergroup Relations, 16, 814-825. doi:10.1177/1368430212471736

*Martins, E., Santos, A. O., \& Colosso, M. (2013). Relações étnico-raciais e Psicologia: Publicações em periódicos da SciELO e Lilacs. Psicologia: Teoria e Prática, 15(3), 118-133.

*Masiero, A. L. (2002). "Psicologia das raças" e religiosidade no Brasil: Uma intersecção histórica. Psicologia: Ciência e Profissão, 22(1), 66-79.

* Masiero, A. L. (2005). A Psicologia racial no Brasil (1918-1929). Estudos de Psicologia (Natal), 10(2), 199-206. doi:10.1590/S1413$-294 X 2005000200006$

*Máximo, T. A. C. O., Larrain, L. F. C. R., Nunes, A. V. L., \& Lins, S. L. B. (2012). Processos de identidade social e exclusão racial na infância. Psicologia em Revista, 18(3), 507-526. doi:10.5752/P.1678-9563.2012v18n3p507

*Mayorga, C., Coura, A., Miralles, N., \& Cunha, V. M. (2013). As críticas ao gênero e a pluralização do feminismo: Colonialismo, racismo e política heterossexual. Revista Estudos Feministas, 21(2), 463-484. doi:10.1590/S0104$-026 \times 2013000200003$

*Naiff, D. G. M., Naiff, L. A. M., \& Souza, M. A. (2009). As representações sociais de estudantes universitários a respeito das cotas para negros e pardos nas universidades públicas brasileiras.
Estudos e Pesquisas em Psicologia, 9(1), 219232.

*Nery, M. P., \& Conceição, M. I. G. (2006). Política racial afirmativa e afetividade na interação intergrupal. Interação em Psicologia, 10(2), 363374.

*Nery, M. P., \& Costa, L. F. (2009). Política afirmativa racial: Polêmicas e processos de identidade do cotista universitário. Psico USF, 14(2), 211220. doi:10.1590/S1413-82712009000200010

Newheiser, A., \& Olson, C. (2012). White and Black American children's implicit intergroup bias. Journal of Experimental Social Psychology, 48(1), 264-270. doi:10.1016/j.jesp.2011.08.011

*Nunes, A. V. L., \& Camino, L. (2011). Atitude político-ideológica e inserção social: Fatores psicossociais do preconceito. Psicologia e Sociedade, 23(1), 135-143. doi:10.1590/S010271822011000100015

*Nunes, S. S. (2006). Racismo no Brasil: Tentativas de disfarce de uma violência explícita. Psicologia USP, 17(1), 89-98.

*Oliveira, C. M. (2002). Pluralidade racial: Um novo desafio para a psicologia. Psicologia: Ciência e Profissão, 22(4), 34-45.

*Oliveira, M. L. P., Meneghel, S. N., \& Bernardes, J. S. (2009). Modos de subjetivação de mulheres negras: Efeitos da discriminação racial. Psicologia e Sociedade, 21(2), 266-274. doi:10.1590/ S0102-71822009000200014

*Oliveira, P., Filho. (2005). Miscigenação versus bipolaridade racial: Contradições e conseqüências opressivas do discurso nacional sobre raças. Estudos de Psicologia (Natal), 10(2), 247-253. doi:10.1590/S1413-294X2005000200012

*Oliveira, P., Filho. (2009). A mobilização do discurso da democracia racial no combate às cotas para afrodescendentes. Estudos de Psicologia (Campinas), 26(4), 429-436. doi:10.1590/S0103$-166 \times 2009000400003$

*Oliveira, P., Filho, Santos, I. O., \& Soares, M. B. (2010). Racialismo e antirracialismo em discursos de estudantes universitários. Revista Psicologia Política, 10(19), 25-40.

Olson, M. A., \& Fazio, R. H. (2009). Implicit and explicit measures of attitudes: The perspective of the MODE model. In R. E. Petty, R. H. Fazio, \& P. Briñol (Eds.), Attitudes, insights from the 
new implicit measures (pp. 19-63). New York: Psychology Press.

*Pereira, C., Torres, A. R. R., \& Almeida, S. T. (2003). Um estudo do preconceito na perspectiva das representações sociais: Análise da influência de um discurso justificador da discriminação no preconceito racial. Psicologia: Reflexão e Crítica, 16(1), 95-107. doi:10.1590/ S0102-79722003000100010

Petruccelli, J. L., \& Saboia, A. L. (2013). Estudos e análises 2 - Informação demográfica e socioeconômica. Características étnico-raciais da população: Classificação e identidades. Rio de Janeiro, RJ: Instituto Brasileiro de Geografia e Estatística.

*Pires, A. M. L. T. (2010). El prejuicio racial en Brasil: medidas comparativas. Psicologia e Sociedade, 22(1), 32-42. doi:10.1590/S010271822010000100005

*Pires, A. M. L. T., \& Alonso, F. M. (2008). El prejuicio racial en Brasil: nuevas medidas y perspectivas. Psicologia e Sociedade, 20(2), 277286. doi:10.1590/S0102-71822008000200015

Raabe, T., \& Beelmann, A. (2011). Development of ethnic, racial, and national prejudice in childhood and adolescence: A multinational meta-analysis of age differences. Child Development, 82(6), 1715-1737. doi:10.1111/j.14678624.2011.01668.x

*Reis, J. T., Filho. (2004). Um perfil do estudante negro da PUC Betim. Psicologia em Revista, 10(15), 139-143. doi:10.5752/203

*Rosemberg, F., Bazilli, C., \& Silva, P. V. B. (2003). Racismo em livros didáticos brasileiros e seu combate: Uma revisão da literatura. Educação e Pesquisa, 29(1), 125-146.

*Roso, A., Strey, M. N., Guareschi, P., \& Bueno, S. M. N. (2002). Cultura e ideologia: A mídia revelando estereótipos raciais de gênero. Psicologia e Sociedade, 14(2), 74-94. doi:10.1590/S010271822002000200005

*Rottenbacher, J. M., Espinosa, A., \& Manuel Magallanes, J. (2011). Analizando el prejuicio: bases ideológicas del racismo, el sexismo y la homofobia en una muestra de habitantes de la ciudad de Lima - Perú. Revista de Psicologia Política, 11(22), 225-246.

*Santos, E. F., \& Scopinho, R. A. (2011). Fora do jogo?: Jovens negros no mercado de trabalho.
Arquivos Brasileiros de Psicologia, 63, 26-37. doi:10.1590/S0104-83332004000200007

*Santos, G. A. (2002). Selvagens, exóticos, demoníacos: Idéias e imagens sobre uma gente de cor preta. Estudos Afro-Asiáticos, 24(2), 275-289. doi:10.1590/S0101-546X2002000200003

*Santos, W. S., Gouveia, V. V., Navas, M. S., Pimente, C. E., \& Gusmão, E. E. S. (2006). Escala de racismo moderno: Adaptação ao contexto brasileiro. Psicologia em Estudo, 11(3), 637-645.

*São Paulo, E. (2010). Preliminary studies on affirmative action in a Brazilian university. $R A M$, Revista de Administração Mackenzie, 11(3), 2745. doi:10.1590/S1678-69712010000300004

*Scandiucci, G. (2006). Cultura hip hop: Um lugar psíquico para a juventude negro-descendente das periferias de São Paulo. Imaginário, 12(12), 225-249.

*Schucman, L. V. (2010). Racismo e antirracismo: A categoria raça em questão. Revista de Psicologia Política, 10(19), 41-55.

*Schucman, L. V. (2014). Sim, nós somos racistas: Estudo psicossocial da branquitude paulistana. Psicologia e Sociedade, 26(1), 83-94. doi:10.1590/S0102-71822014000100010

*Serra, L. N., \& Schucman, L. V. (2012). Branquitude e progresso: A Liga Paulista de Higiene Mental e os discursos paulistanos na contemporaneidade. Estudos e Pesquisas em Psicologia, 12(1), 288-311.

*Silva, M. P. (2001). O anti-racismo no Brasil: Considerações sobre o estatuto social baseado na consciência racial. Revista Psicologia Política, 1(1), 37-65.

*Silva, P. V. B., Rocha, N. G., \& Santos, W. O. (2012). Negras(os) e brancas(os) em publicidades de jornais paranaenses. Intercom, Revista Brasileira de Ciências da Comunicação, 35(2), 149-168. doi:10.1590/S1809-58442012000200008

*Silva, P. V. B., Teixeira, R., \& Pacifico, T. M. (2013). Políticas de promoção de igualdade racial e programas de distribuição de livros didáticos. Educação e Pesquisa, 39(1), 127-143. doi:10.1590/S1517-97022013000100009

*Silva, S. G. (2003). Preconceito no Brasil contemporâneo: As pequenas diferenças na constituição das subjetividades. Psicologia: Ciência e Profissão, 23(2), 2-5. doi:10.1590/S141498932003000200002 
Sritharan, R., \& Gawronski, B. (2010). Changing implicit and explicit prejudice: Insights from the Associative-Propositional Evaluation Model. Social Psychology, 41, 113-123. doi:10.1037/0022-3514.87.1.5

*Tavares, N. O., Oliveira, L. V., \& Lages, S. R. C. (2013). A percepção dos psicólogos sobre o racismo institucional na saúde pública. Saúde em Debate, 37(99), 580-587. doi:10.1590/S010311042013000400005

Telles, E. E. (2003). Race in another America: The significance of skin color in Brazil. Princeton, NJ: Princeton University Press.

*Teshainer, M. C. R., \& Küller, A. L. M. (2005). Por que o desdém: Reflexões sobre o racismo. Psicologia Revista, 14(2), 257-278.

*Vasconcelos, T. C., Gouveia, V. V., Souza, M. L., Filho, Sousa, D. M. F., \& Jesus, G. R. (2004). Preconceito e intenção em manter contato social: Evidências acerca dos valores humanos. Psico USF, 9(2), 147-154.

*Vianna, P. C., \& Neves, C. E. A. B. (2011). Dispositivos de repressão e varejo do tráfico de drogas: Reflexões acerca do Racismo de Estado. Estudos de Psicologia (Natal), 16(1), 31-38.
*Vilhena, J. A. (2006). A violência da cor: Sobre racismo, alteridade e intolerância. Revista Psicologia Politica, 6(12). Recuperado em http:// www.fafich.ufmg.br/rpp/seer/ojs/viewarticle. php?id=7\&layout $=\mathrm{html}$

*Weyler, A. R. (2006). A loucura e a República no Brasil: A influência das teorias raciais. Psicologia USP, 17(1), 17-34. doi:10.1590/S010365642006000100003

*Zamora, M. H. R. N. (2012). Desigualdade racial, racismo e seus efeitos. Fractal, Revista de Psicologia, 24(3), 563-578. doi:10.1590/S198402922012000300009

Zoltowski, A. P. C., Costa, A. B., Teixeira, M. A. P., \& Koller, S. H. (2014). Qualidade metodológica das revisões sistemáticas em periódicos de Psicologia brasileiros. Psicologia: Teoria e Pesquisa, 30(1), 97-104. doi:10.1590/S010237722014000100012 